\section{J. RIQUET, F. PITEL}

INRA, Laboratoi re de Généti que Cellulai re, BP 27, 31326 Castanet-Tolosan cedex

e-mail : riquet@toulouse.inra.fr

\title{
Les techniques de
base de la génétique \\ Les techniques de
base de la génétique moléculaire
}

Rés umé. Les techni ques les plus couramment uti li sées en généti quemoléculai re font l'objet de cet arti cle La premi ère parti e présente les outils de base, fondés sur des pri ncipes de physi que, de chi mi e ou de bi ologi e. Di fférentes combi nai sons de ces outi ls sont ensui te développées pour aborder les techni ques uti li sées dans différents domai nes de la bi ologi e moléculai re : fragmentati on, séparation, repérage, multi pli cati on et lecture de l'ADN. L'accent est mi s sur la di versi té des techni ques pouvant être mi ses en œuvre et sur la vi tesse d'évoluti on de ces techni ques.

Cet article constitue un préambule à ceux concernant l'utilisation des techniques moléculaires dans nos domaines de recherches. Les méthodes de biologie moléculaire ne sont pas toutes évoquées, seules les plus couramment utilisées pour l'analyse de l'ADN sont présentées. Leurs limites sont liées à chaque type d'utilisation qui en est faite, les avantages et inconvénients de chaque technique seront donc évoqués dans les autres articles de cet ouvrage.

Les techniques de génétique moléculaire reposent sur un nombre limité d'outils (hybridation, électrophorèse, enzymes, cellules ...), qui seront présentés avant de détailler les techniques principales de manipulation de l'ADN.

Les techniques mises au point pour analyser I'ADN sont tributaires de la constitution même des génomes. Le nombre de paires de bases d'un génome de vertébré dépasse en général le milliard $\left(1,2.10^{9}\right.$ paires de bases chez la poule, $3.10^{9} \mathrm{chez}$ les mammifères). La biologie moleculaire appliquée à la génétique a pour but l'étude d'une région du génome et la recherche d'un gène, voire d'une mutation (concernant, à la limite, une seule base parmi les quelques milliards). Les techniques mises au point ont donc pour objectif de cibler le fragment intéressant au sein d'une molécule d'ADN des millions de fois plus grande. Le facteur «quantité» est le plus souvent limitant : la quantité d'ADN contenue dans une cellule est infime (de l'ordre du picogramme). II faut donc également disposer de techniques permettant l'amplification préalable du matériel que I'on souhaite étudier.

Les recherches menées sur l'ARN reposent pour une grande part sur des méthodes similaires. En revanche les protéines ont des caractéristiques particulières, et une partie seulement des techniques présentées peuvent leur être appliquées. Nous avons choisi de ne pas évoquer ici les procédés propres aux études d'expression des gènes faisant intervenir ARN et protéines.

\section{1 / Les outils de la biologie moléculaire en génétique}

Les techniques de base de la biologie moléculaire consistent le plus souvent à tirer parti des caractéristiques propres du matériel biologique sur lequel on travaille (comme les propriétés physico-chimiques de l'ADN) et à exploiter des organismes, surtout des microorganismes, du monde vivant.

\section{1 / Propriétés de l'ADN}

L'ADN est une molécule double brin, dont les deux brins sont complémentaires. Si on sépare les deux brins (par simple chauffage ou par action d'un agent dénaturant comme la soude, l'urée ou la formamide), on a donc accès à deux molécules simples brins. Un fragment d'ADN plus ou moins long, simple brin et de séquence complémentaire à la séquence cible pourra s'associer à celle-ci : il y a hybridation de deux brins complémentaires. Des techniques comme la PCR (Polymerase Chain Reaction), les Southern blots, le criblage de banques et l'hybridation in si tu s'appuient sur cette caractéristique.

L'ADN est constitué de nucléotides, auxquels une modification peut être apportée (isotopes radioactifs du phosphore ou du soufre, molécule fluorescente). Ces constituants modifiés de l'ADN permettent alors son marquage et la visualisation de la molécule.

L'ADN est une molécule chargée négativement. Cette propriété permet de faire migrer de I'ADN sur différents supports dans un champ électrophorétique.

L'ADN a d'autres propriétés physico-chimiques que nous n'aborderons pas, et qui permettent de lui faire subir sonications (fragmentation aléatoire à l'aide d'ultra-sons), centrifugations, dosages, précipitations, chromatographies et autres manipulations dont le détail se trouve dans tout bon ouvrage de biologie moléculaire ( $\mathrm{cf}$ liste des références). 


\section{2 / Biologie moléculaire}

La génétique moléculaire fait appel à des manipulations de molécules organiques, qui sont à l'origine synthétisées, digérées, liées, détruites in vivo. Les outils de la biologie moléculaire ont donc été en partie fournis par le matériel vivant lui-même.

L'ADN est présent dans tous les tissus animaux, et les manipulations sur cette molécule nécessitent au préalable son extraction. Le tissu le plus souvent utilisé comme source d'ADN est le sang : on peut I'obtenir facilement (sans opération invasive), et il contient une grande quantité d'ADN contrairement à d'autres sources possibles d'ADN (poils, plumes...). Les techniques d'extraction sont nombreuses (choisies en fonction de la quantité de matériel de départ, de la source d'ADN, de la qualité requise...), et font appel à des manipulations successives (lyse, précipitation, centrifugation...).

La culture in vitro de cellules est un préalable à de nombreuses applications de biologie moléculaire (hybridation in situ, études d'expression de gènes, fabrication d'hybrides somatiques...).

Les microorganismes tels que des bactéries ou des levures sont utilisés dans les opérations de manipulation in vi tro de fragments d'ADN.

En outre, à partir des bactéries, on a isolé des molécules capables de reproduire in vitroles transformations de I'ADN qu'elles effectuent naturellement in vivo: les enzymes. Elles représentent une bonne part des outils de base pour le biologiste moléculaire.

Les enzymes de restriction reconnaissent un site spécifique de l'ADN (souvent 4 ou 6 paires de bases palindromiques), et hydrolysent la molécule dans ce site ou au voisinage de celui-ci. Elle permettent d'obtenir, à partir d'une molécule entière, des fragments d'ADN dont la longueur et le nombre vont dépendre de la répartition des sites de restriction sur le fragment d'origine. La coupure peut générer des bouts francs ou des bouts cohésifs (figure 1). Elles sont utilisées dans de nombreuses techniques de biologie moléculaire, comme les Southern blots, le clonage, la recherche de certains polymorphismes, etc.

Les polymérases synthétisent le brin complémentaire d'un brin d'ADN à partir de nucléotides libres présents dans le milieu réactionnel. La Taq polymérase (isolée de la bactérie Thermophi lus aquati cus), thermostable, est utilisée notamment dans la réaction PCR. Le fragment Klenow de I'ADN polymérase I (extraite de la bactérie Escherichia coli) est employé pour le marquage de l'ADN.

Les ligases assemblent des fragments d'ADN linéaires entre eux. Elles créent une liaison covalente entre les extrémités, de séquences compatibles, de deux fragments d'ADN (figure 1). Elles sont utilisées notamment dans les procédures de clonage, dans l'addition d'adaptateurs (oligonucléotides de séquence connue) à de l'ADN génomique digéré, dans certaines analyses de polymorphisme ...

D'autres enzymes sont très utiles en biologie moléculaire, comme les kinases (qui ajoutent un groupement phosphate en 5'), les phosphatases (qui, à l'inverse, éliminent le groupement phosphate), et d'autres encore (RNAses, DNA ses, nickases...) dont nous ne pouvons ici faire l'inventaire exhaustif.

\section{2 / Les techniques de manipulation de I'ADN}

La plupart des techniques utilisées en génétique moléculaire sont mises au point en combinant les outils de base précédemment décrits. Les techniques utilisées peuvent être classées selon leurs objectifs, à savoir fragmenter l'ADN, séparer les fragments, repérer les morceaux d'ADN d'intérêt puis multiplier le fragment à étudier et, enfin, à l'échelle la plus petite, lire directement la séquence d'ADN.

Figure 1. Exemples d'enzymes de restriction utilisées en génétique moléculaire : une digestion par l'enzyme Haelll génère des extrémités franches, Sau3Al et BamHI des extrémités cohésives 5' sortantes et compatibles entre elles, l'enzyme Pst/ des extrémités 3' sortantes. Certaines enzymes comme Mboll digèrent l'ADN en dehors de leur site de reconnaissance.

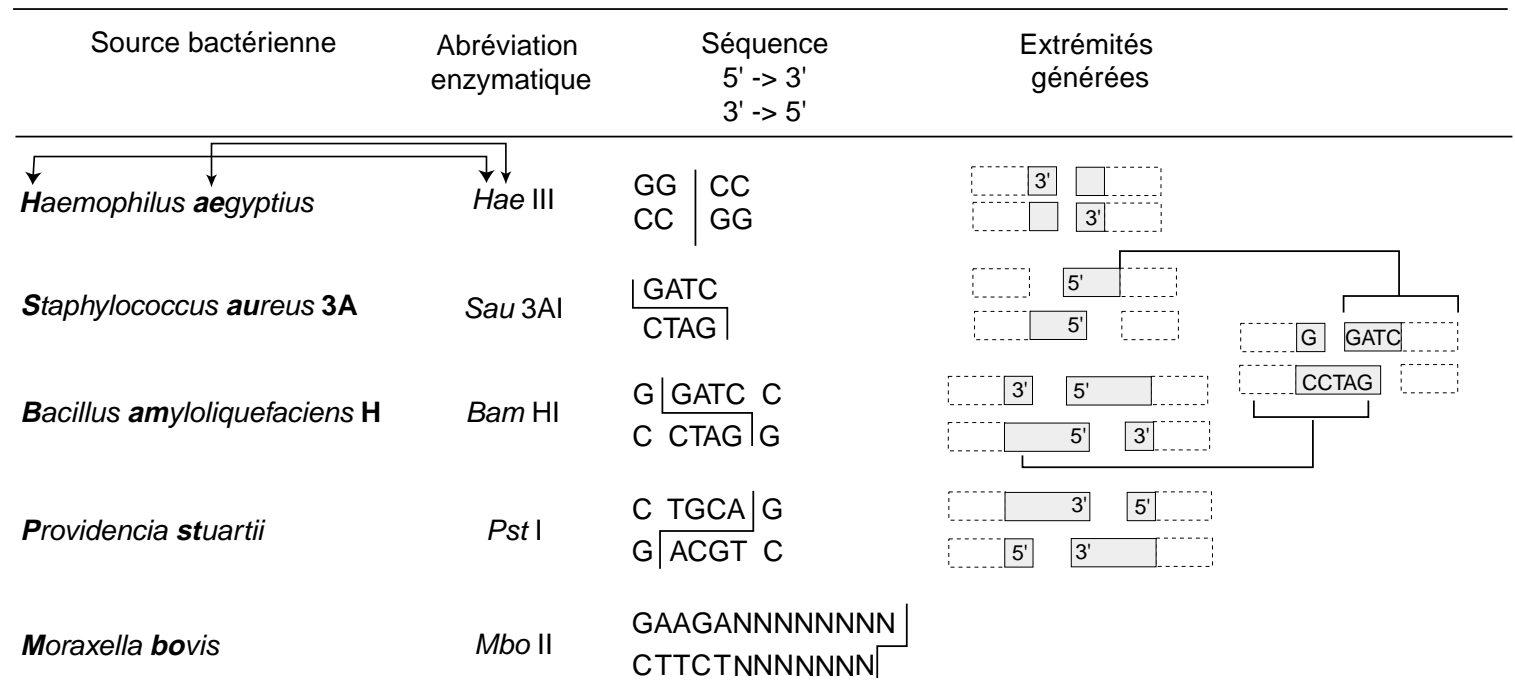




\section{1 / F ragmenter}

Les méthodes d'analyse ne permettent pas d'appréhender globalement la totalité de I'ADN. Toute manipulation nécessite donc une fragmentation préalable du génome :

- tirer parti de la condensation de I'ADN en chromosomes lors de la métaphase est le premier moyen de travailler sur un génome fragmenté. Ce phénomène est exploité dans les techniques d'hybridation in situ sur chromosomes (Yerle 2000, cet ouvrage) et dans les manipulations de tri de chromosomes (voir plus loin) ;

- on peut également s'intéresser uniquement à la partie codante de l'ADN (5 à $10 \%$ du génome) en ne travaillant que sur les $A D N$ complémentaires $(A D N c)$, c'est-à-dire les fragments d'ADN synthétisés à partir des ARN messagers (ARNm) présents dans les tissus étudiés ;

- nous avons la possibilité, grâce aux enzymes de restriction, de fractionner la molécule d'ADN pour obtenir des fragments dont la taille est compatible avec l'utilisation des techniques de laboratoire. D'autres types de fragmentation sont également utilisés (sonication, fragmentation mécanique, irradiation...) ;

- une manière de «fragmenter» I'ADN est de ne cibler dès le départ qu'une petite portion du génome : l'utilisation de la PCR (voir plus loin) entre dans ce cadre.

\section{2 / Séparer}

La molécule d'ADN étant fragmentée, il faut séparer les différents fragments pour les visualiser individuellement ou pour sélectionner ceux qui nous intéressent.

\section{Pour voir}

L'une des techniques appartenant au quotidien du biologiste moléculaire est l'électrophorèse. Elle permet de séparer les fragments d'ADN en fonction de leur taille : la migration dans un champ électrique est effectuée sur différents types de gels - dans lesquels la mobilité est influencée par la densité du maillage moléculaire - avec différentes conditions de migration, qui peuvent affecter la mobilité des fragments.

Gels d'agarose: en fonction du pourcentage d'agarose dans le gel, des fragments de quelques dizaines à quelques milliers de paires de bases peuvent être discriminés, en champ électrique constant. Ces gels sont utilisés couramment pour séparer des fragments d'ADN avant Southern blot (voir plus loin), vérifier la qualité ou la quantité d'un échantillon d'ADN, déterminer la taille d'un produit PCR, d'une insertion d'ADN dans un vecteur... Grâce à l'utilisation de champs électriques alternatifs, on peut aller jusqu'à la séparation de fragments de plus de 10000 kilobases : c'est l'électrophorèse en champs pulsés.

Gels d'acrylami de: ils sont plus résolutifs que les gels d'agarose et permettent de séparer des fragments de quelques dizaines à quelques centaines de paires de bases, à la base près. Ils sont utilisés tels quels pour la séparation fine de fragments d'ADN de tailles voisines : séquençage d'ADN, analyses de polymorphismes de longueur comme les microsatellites ou les AFLP ... Différentes compositions de ces gels ( par exemple présence ou absence d'agents dénaturants comme la formamide ou l'urée), associées à différentes conditions de migration (à chaud, à froid, en gradient de température...) donnent autant de techniques différentes qui permettent de révéler des polymorphismes de toutes sortes.

\section{Pour cibler}

Dans certaines conditions, l'ADN est individualisé en chromosomes. Si les chromosomes d'une espèce sont suffisamment différents entre eux (chromosomes de porc ou de bovins, macrochromosomes de poule), ils peuvent être triés, par cytométrie en flux, en fonction de leur taille et de leur composition en paires de bases. On peut ainsi isoler plusieurs centaines de copies d'un chromosome qui nous intéresse, pour diverses applications comme le «chromosome-painting» en cartographie comparée (Gellin 2000, cet ouvrage) ou la recherche de marqueurs spécifiques d'un chromosome.

La fabrication d'hybrides somatiques (Yerle 2000, cet ouvrage) permet de disposer de chromosomes ou fragments de chromosomes séparés dans des clones différents.

La technique la plus courante permettant de cibler une portion du génome est le clonage (figure 2) : I'ADN génomique est fragmenté à l'aide d'enzymes de restriction et inséré dans un «vecteur». Ce vecteur permet alors l'introduction et la multiplication de la séquence dans un microorganisme hôte (bactérie ou levure). Chaque cellule hôte intégrera un fragment d'ADN différent et l'ensemble de ces clones constituera une «banque» du génome étudié.

En fonction du matériel à cloner (ADN génomique, $A D N C$ ), de l'utilisation ultérieure que l'on veut faire de la banque (criblage, séquençage, expression...), de la taille des fragments que l'on veut obtenir (quelques centaines de paires de bases ou quelques kilobases), on utilise l'un ou l'autre des vecteurs de clonage existants : plasmide ( $100 \mathrm{pb}$ à 5 $\mathrm{kb})<$ phage (10 à $20 \mathrm{~kb}$ ) < cosmide (environ $40 \mathrm{~kb}$ ) $<$ PAC ou BAC (50 à $200 \mathrm{~kb})<$ YAC (100 à $2000 \mathrm{~kb}$ ) (Rogel-Gaillard 2000, cet ouvrage).

\section{3 / Repérer}

En dehors de l'utilisation de la microscopie (par exemple, visualisation de chromosomes en métaphase en cytogénétique) ou lorsque l'ADN est précipité sous forme de «méduse» au cours d'extractions de grandes quantités d'ADN, cette petite molécule n'est pas visible.

Plusieurs méthodes de révélation de I'ADN sont disponibles, utilisées seules ou en combinaison.

\section{Par coloration}

Deux méthodes de coloration de I'ADN sans marquage préalable sont couramment utilisées:

- la révélation par bromure d'éthidium (BET) : cet agent intercalant se fixe à l'ADN double brin, et émet une fluorescence quand il est excité par des rayons ultraviolets ;

- la coloration à l'argent : cette technique est le plus souvent utilisée pour les colorations de fragments simples brins, pour lesquels le BET est moins efficace. Elle est basée sur le même principe que la photographie et permet la formation d'un précipité d'argent sur la molécule d'ADN. 
Figure 2. Principe de construction d'une banque plasmidique d'ADN génomique total. Les éléments du vecteur essentiels au clonage sont indiqués : l'origine de réplication permet la multiplication du vecteur dans la cellule hôte de manière indépendante de la bactérie ; le gène de résistance à un antibiotique permet de sélectionner les bactéries ayant intégré un vecteur (la bactérie est, quant à elle, sensible à l'antibiotique utilisé dans le milieu de culture des boîtes) ; le multisite de clonage permet l'insertion de l'ADN étranger ; cette séquence se trouve au milieu du fragment du gène LacZ, ce qui permet une discrimination par la couleur des bactéries dont le vecteur contient un insert.
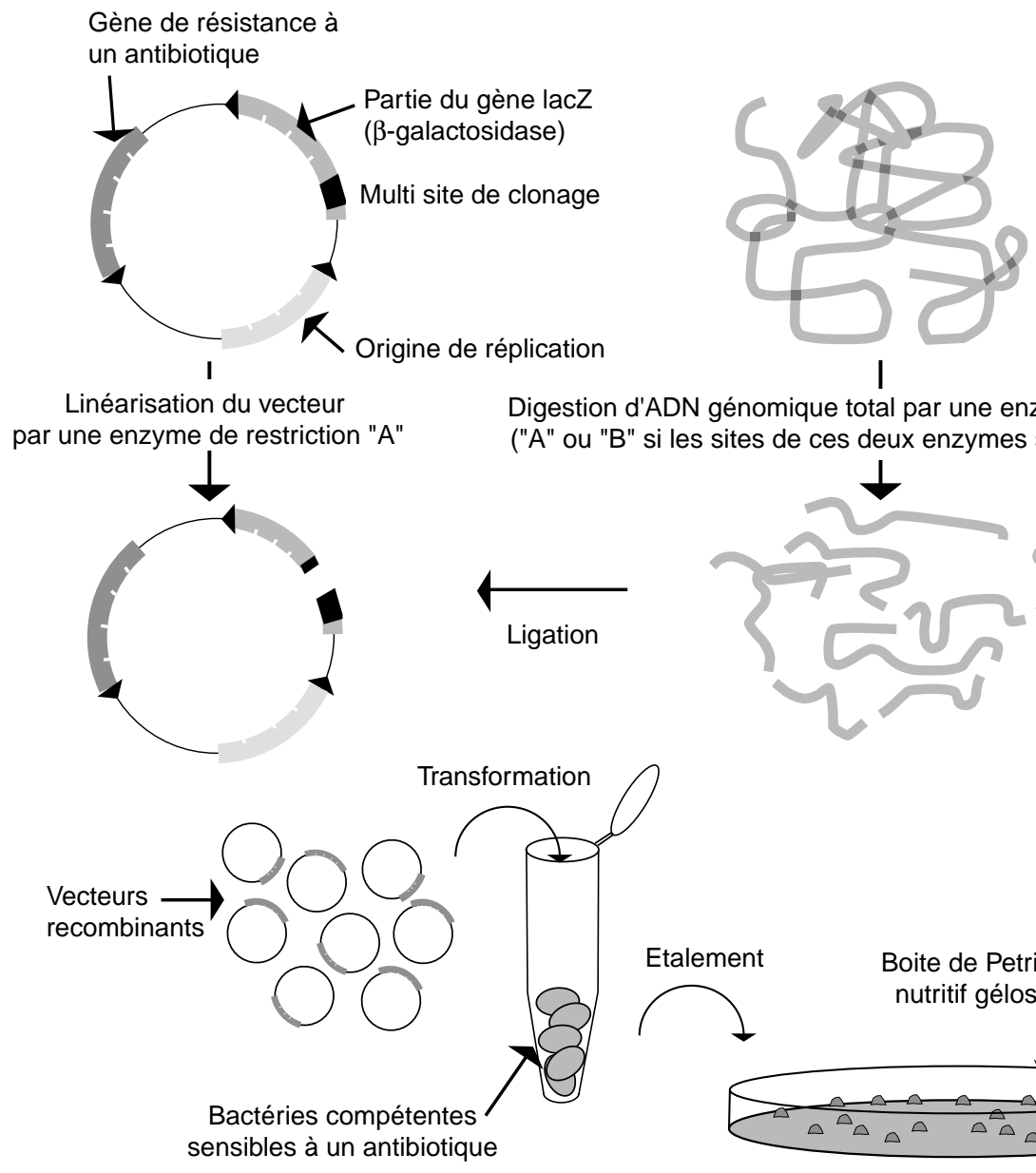

Etalement Boite de Petri contenant un milieu

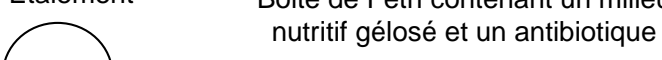

\section{Par marquage}

Au lieu d'être révélés après leur séparation, les fragments d'ADN peuvent être marqués au préalable : - par des éléments radioactifs: ${ }^{32} \mathrm{P},{ }^{33} \mathrm{P}$ et ${ }^{35} \mathrm{~S}$ sont les radioéléments les plus utilisés en biologie moléculaire. II faut leur ajouter le tritium, utilisé en hybridation in situ. Le principe du marquage d'un fragment d'ADN est l'incorporation, grâce à une enzyme, de nucléotides marqués radioactivement. Sans entrer dans les détails, cette incorporation peut être effectuée au sein même de la molécule («nick-translation» ou «random-priming», avec utilisation du fragment Klenow de l'ADN polymérase I), à son extrémité $3^{\prime}$ (terminale transférase), ou à son extrémité 5' (polynucléotide kinase). L'ADN est révélé par simple autoradiographie. Cette méthode est longtemps restée la technique prépondérante pour la révélation de fragments d'ADN. Certains lui préfèrent actuellement le marquage par des produits non radioactifs comme la biotine ou la digoxigénine (suivi de méthodes de détection spécifiques) ;

- par des fluorophores : une des manières de visualiser un fragment d'ADN est de le marquer par un fluorophore préalablement à la migration. L'excitation de ce fluorophore par un laser entraîne l'émission de fluorescence, détectée par une caméra.
C'est le principe du séquenceur automatique, très utilisé pour le séquençage et le typage de marqueurs génétiques comme les microsatellites. Les amorces servant à l'amplification PCR ou à la réaction de séquence sont marquées, de manière à détecter en temps réel le fragment obtenu, pendant son passage devant le laser. On peut également incorporer des nucléotides marqués pendant la réaction PCR.

L'utilisation d'un séquenceur a de nombreux avantages par rapport à des migrations sur gel classique suivies de révélation. Citons notamment la possibilité de déposer plusieurs échantillons dans un même puits (quatre fragments différents de même taille pourront être distingués à l'aide de quatre fluorophores différents), la lecture immédiate des résultats et, surtout, l'informatisation des données, qui permet des gains considérables de temps et de fiabilité des résultats.

\section{Par hybridation d'une sonde marquée}

Les techniques précédemment décrites s'intéressent à la révélation de fragments d'ADN que l'on souhaite visualiser directement (après PCR, électrophorèse de fragments discernables ...). Elles s'appliquent également au marquage des sondes utilisées en hybridation : dans de nombreux cas, on ne s'in- 
Figure 3. Principe du criblage d'une banque plasmidique d'ADN génomique total. Une membrane de nylon est posée sur la boîte de Pétri (1 et 2). Une partie des bactéries de chaque colonie se fixe à la membrane (3). La réplique bactérienne, après dénaturation de l'ADN, est hybridée à une sonde radioactive (4). Après lavages (élimination de l'excès de sonde), un film autoradiographique est mis en contact de la membrane (5). Les "spots positifs" correspondent aux clones contenant un fragment d'ADN homologue de la sonde (6).

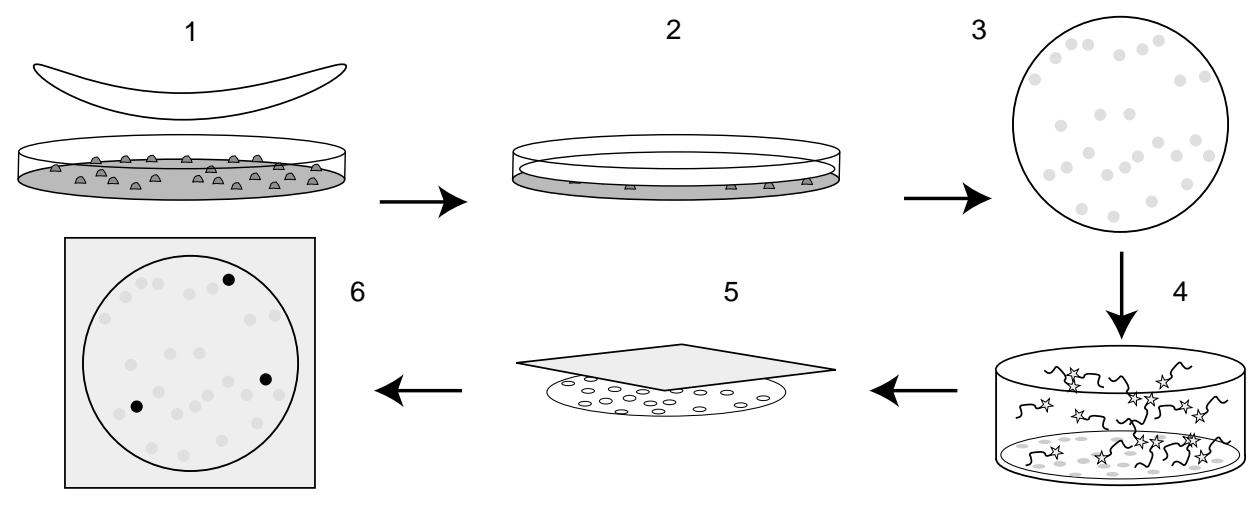

téresse qu'à une région précise du génome, perdue dans un mélange complexe contenant la totalité de I'ADN. On a alors recours à l'hybridation. La complémentarité des deux brins d'ADN permet de récupérer le fragment intéressant dans le génome grâce à l'hybridation sur la molécule d'ADN d'une sonde spécifique : cette sonde est une courte séquence d'ADN (de quelques dizaines de paires de bases à quelques kilobases), dont la séquence est suffisamment complémentaire de l'ADN cible pour permettre une hybridation spécifique. Une sonde marquée permet l'observation, voire la sélection, du fragment intéressant.

Différents types de sondes (uniques, répétées, génomiques, ADNc) et différentes sortes d'hybridation (sur chromosomes en métaphase, colonies bactériennes, membranes ...) peuvent être combinées en fonction de l'objectif à atteindre :

- pour connaître la localisation d'un gène sur un chromosome, on utilisera une sonde correspondant à un fragment de ce gène (génomique ou $A D N C$ ), que I'on hybridera sur les chromosomes en métaphase. La sonde fluorescente ou radioactive sera ensuite repérée à l'endroit où elle s'est hybridée sur le chromosome ;

- pour sélectionner, dans une banque d'ADN génomique d'une espèce, un clone portant le fragment du gène intéressant, mais pour lequel on ne dispose que d'une sonde hétérologue (gène humain à isoler chez la poule par exemple), on effectuera l'hybridation sur des membranes où l'ADN des clones d'une banque de l'espèce étudiée aura été préalablement fixé (répliques bactériennes). La sonde étant marquée (radioactivement par exemple), on visualisera les colonies sur lesquelles la sonde s'est hybridée: c'est le criblage de la banque (figure 3 ) ;

- pour isoler, à partir d'une banque, des clones portant un microsatellite, on pourra hybrider les répliques bactériennes avec une sonde marquée dont la séquence correspondra au motif répété des marqueurs que l'on souhaite isoler, $(C A)_{10}$ par exemple.

On peut également hybrider une sonde sur un Southern blot: I'ADN génomique est digéré, les fragments sont séparés en fonction de leur longueur par électrophorèse. Les fragments d'ADN sont ensuite dénaturés in si tu, pour être transférés à l'état simple brin sur membrane. Ces fragments sont alors mis en présence d'une sonde, généralement radioactive, ce qui permet après révélation autoradiographique d'obtenir un profil de bandes spécifiques. Cette technique est utilisée pour l'analyse de marqueurs génétiques (RFLP ou minisatellites), pour des comparaisons de profil d'hybridation d'un fragment de gène sur différentes espèces (Zooblot) ... Si la membrane n'est pas issue d'un gel, mais du dépôt sous forme de points de produits PCR, on parle de dot-blot.

Actuellement, les programmes systématiques d'étude des génomes ont favorisé la mise en place de filtres à haute densité pour des hybridations en grand nombre: les produits PCR ou les clones bactériens sont déposés de façon dense et régulière sur membranes, voire sur micro-support (puces à ADN : Rogel-Gaillard 2000, Hatey 2000, cet ouvrage).

\section{4 / Multiplier}

L'ADN génomique étant une denrée rare, la première façon d'en obtenir des quantités importantes est d'utiliser la multiplication naturelle de cette molécule, c'est-à-dire de l'extraire à partir d'un nombre important de cellules (extractions à partir de sang, culture de cellules en grande quantité pour les extractions à partir d'hybrides somatiques, etc). Dans un deuxième temps, quand on s'intéresse à un fragment particulier du génome, il existe plusieurs possibilites pour l'amplifier.

\section{Clonage}

Le clonage, déjà évoqué plus haut dans l'isolement de fragments d'ADN, permet d'obtenir en grande quantité le fragment d'ADN cloné dans une colonie bactérienne : une sonde spécifique, un fragment d'ADN génomique à séquencer, un ADN c, etc, peuvent être conservés et multipliés par la bactérie grâce à la multiplication cellulaire. Le vecteur porté par la bactérie se multiplie en parallèle à I'ADN bactérien, et une extraction spécifique permet ensuite d'isoler l'ADN intéressant de l'ADN de la bactérie.

\section{PCR}

La PCR (Polymerase Chain Reaction) est, depuis sa mise au point (Saiki et al 1985), la technique de base de la biologie moléculaire : elle permet à la fois de cibler le ou les fragments que l'on veut étudier, et d'augmenter leur quantité de manière exponentielle (figure 4). Cette technique a pourtant deux limites : d'une part un minimum d'information sur la séquence est nécessaire pour la définition d'amorces spé- 
Figure 4. Principe de la PCR (Polymerase Chain Reaction). Chaque cycle est décomposé en trois étapes : dénaturation des deux brins d'ADN, hybridation spécifique des amorces encadrant le fragment à amplifier et élongation (synthèse des brins complémentaires par une polymérase). A chaque cycle, il y a doublement $d u$ nombre de copies du fragment amplifié, les brins néosynthétisés auparavant servant également de matrice. Une réaction de 30 cycles d'amplification produit en théorie $2^{30}$ copies du fragment.

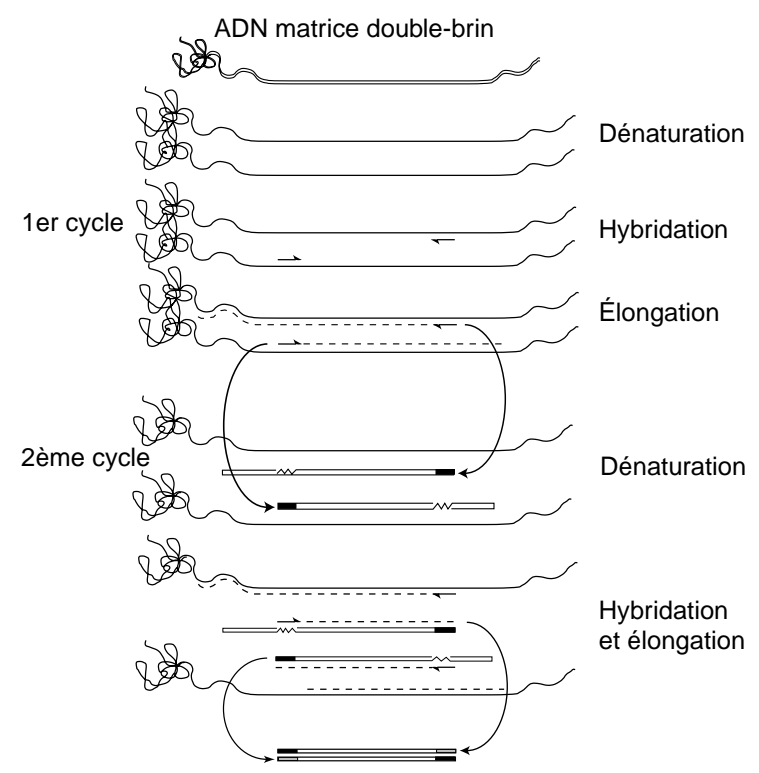

cifiques permettant l'amplification ciblée d'un fragment et, d'autre part, la taille maximum des fragments est limitée par l'efficacité des polymérases utilisées (de quelques paires de bases à une vingtaine de kilobases). Elle est utilisée dans tous les types d'application.

\section{5 / Lire}

Le stade ultime de la biologie moléculaire est de travailler directement à l'échelle de la paire de bases.

\section{Séquençage}

On peut actuellement accéder à la séquence d'un fragment d'ADN grâce au séquençage (figure 5).

L'utilisation de fluorophores a permis de simplifier ce protocole. Les améliorations apportées au cours des dernières années à la chimie de ces molécules et l'utilisation de nouvelles polymérases, font que ce protocole est utilisé actuellement dans le cadre de programmes de séquençage systématique des génomes. La mise en place, à grande échelle, de ce type de programme en génomique a également favorisé le développement d'une nouvelle technique de séquençage sur puces à ADN. Elle consiste à hybrider le fragment à séquencer sur des oligonucléotides (représentant toutes les combinaisons de bases possibles) fixés sur microsupports. L'intensité des signaux d'hybridation obtenus permet de déduire la séquence du fragment.

Figure 5. Principe du séquençage. Cette technique repose sur l'utilisation de di-désoxynucléotides, qui stoppent la réaction de synthèse. Le fragment à séquencer est dénaturé et une seule amorce est hybridée sur l'ADN matrice. Une polymérase synthétise le brin complémentaire à l'aide de nucléotides présents dans le mélange. L'incorporation aléatoire d'un di-désoxynucléotide stoppe la synthèse du fragment. Le produit obtenu correspond donc à un mélange de fragments de tailles différentes : à chaque base de la matrice d'origine correspond un ensemble de fragments de même taille. La visualisation des fragments après électrophorèse permet de déduire la séquence recherchée.

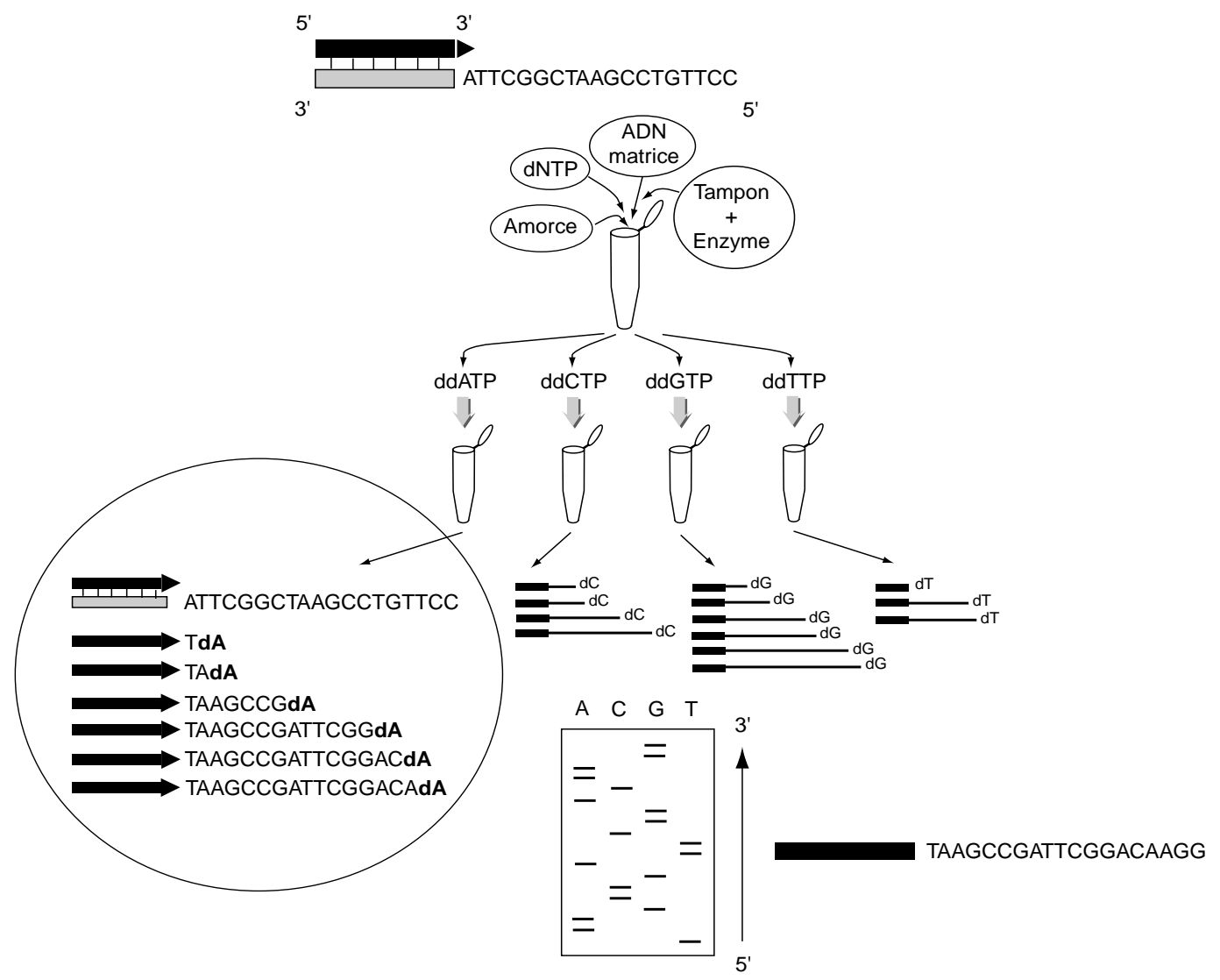


Cette technique est actuellement utilisée en génétique humaine pour le séquençage systématique du virus HIV et de gènes impliqués dans certains cancers (recherche de mutants permettant un traitement plus ciblé). En cartographie génétique, cette nouvelle technologie est par ailleurs utilisee pour le génotypage systématique de marqueurs bialléliques (Pitel et Riquet 2000, cet ouvrage).

\section{Conclusion et perspectives}

En fonction de l'origine du matériel sur lequel on travaille, de l'objectif à atteindre et de l'échelle du programme à mener à bien, différentes techniques de biologie moléculaire sont utilisées. Toutes reposent sur un ensemble d'outils de base à peu près immuable. En revanche, les techniques elles-mêmes évoluent très vite, et certaines de celles qui ont été présentées ici ne seront sans doute plus utilisées que de manière ponctuelle dans quelques années.

La volonté de travailler à grande échelle sur le génome humain (séquençage complet, criblage des mutations d'un gène entier ... ) a amené la biologie moléculaire à évoluer vers des techniques plus performantes, avec par exemple la miniaturisation des outils pour des manipulations d'un très grand nombre d'échantillons en simultané (mise au point des puces à $A D N$ ). La génétique moléculaire chez les animaux domestiques a toujours bénéficié des avancées de la génétique humaine. Nous sommes probablement à l'orée d'un changement d'échelle de nos programmes du fait de l'emploi d'un nouveau type d'outils générant une masse importante de données et de l'utilisation systématique de l'informatique.

\section{Références}

Gellin J ., 2000. La cartographie comparée des génomes des vertébrés. INRA Productions Animales, numéro hors série «Génétique moléculaire : principes et application aux populations animales », 95-102.

Hatey $F ., 2000$. Recherche de gènes associés à des fonctions : I'approche fonctionnelle. INRA Productions Animales, numéro hors série «Génétique moléculaire : principes et application aux populations animales », 153-160.

Kaplan J.C., Delpech M., 1994. Biologie moléculaire et médecine (2ème Ed). Flammarion Médecines-Sciences, Paris.

Pitel F., Riquet J ., 2000. Les marqueurs anonymes et la détection de leur polymorphisme. INRA Productions Animales, numéro hors série «Génétique moléculaire : principes et application aux populations animales », 45-53.

Rogel-Gaillard C., 2000. Les banques de grands fragments d'ADN. INRA Productions Animales, numéro hors série « Génétique moléculaire : principes et application aux populations animales », 79-85.
Saiki R.K., Scharf S., Faloona F., Mullis K.B., Horn G.T., Erlich H.A., Arnheim N., 1985. Enzymatic amplification of beta-globin genomic sequences and restriction site analysis for diagnosis of sickle cell anemia. Science, 230, 1350-1354.

Sambrook J ., Fritsch E.F., Maniatis T., 1989. Molecular cloning, a laboratory manual (2nd Ed). Cold Spring Harbor Laboratory Press.

Strachan T., Read A.P., 1998. Génétique moléculaire humaine. Flammarion Médecine-Sciences, Paris.

Tagu D., 1999. Principes des techniques de biologie moléculaire. INRA Editions, Paris.

Yerle M., 2000. Etablissement des cartes cytogénétiques et physiques. INRA Productions Animales, numéro hors série "Génétique moléculaire : principes et application aux populations animales », 87-93. 
